\title{
Balanced-detection Raman-induced Kerr-effect spectroscopy
}

\author{
Vikas Kumar, ${ }^{1}$ Michele Casella, ${ }^{1}$ Egle Molotokaite, ${ }^{1}$ Davide Gatti, ${ }^{1}$ Philipp Kukura, ${ }^{2}$ \\ Cristian Manzoni, ${ }^{1}$ Dario Polli, ${ }^{1}$ Marco Marangoni, ${ }^{1}$ and Giulio Cerullo ${ }^{1}$ \\ ${ }^{1}$ Institute for Photonics and Nanotechnologies, Consiglio Nazionale delle Ricerche, Dipartimento di Fisica, \\ Politecnico, Piazza L. da Vinci 32, 20133 Milano, Italy \\ ${ }^{2}$ Physical and Theoretical Chemistry Laboratory, Oxford University, South Parks Road, Oxford OX1 3QZ, United Kingdom
}

(Received 27 July 2012; revised manuscript received 26 September 2012; published 9 November 2012)

\begin{abstract}
We introduce balanced-detection (BD) Raman-induced Kerr-effect (RIKE) as a powerful coherent Raman scattering (CRS) technique. RIKE relies on the Raman-induced birefringence that occurs when the pump-Stokes frequency detuning is in resonance with a vibrational transition. We show how a balanced-detection configuration, inspired by that applied in electro-optic sampling, suppresses both the linear and nonlinear backgrounds that affect other CRS techniques. BD-RIKE enables homodyne amplification of the signal and linear dependence on the concentration of the target oscillators, as well as cancellation of laser intensity noise. In addition, it allows straightforward selection of either the imaginary or the real part of the nonlinear vibrational response.
\end{abstract}

DOI: 10.1103/PhysRevA.86.053810

PACS number(s): 42.65.Dr, 42.62.Fi, 42.65.Hw, 78.47.nj

\section{INTRODUCTION}

Coherent Raman scattering (CRS) encompasses a powerful suite of techniques for chemically sensitive identification of molecules on the basis of their characteristic vibrational frequencies [1]. CRS is breaking ground in biomedical optics, thanks to its capability of noninvasive, label-free imaging of tissues and cells [2-5], as well as in remote sensing of pollutants and hazardous molecular compounds [6]. With respect to spontaneous Raman scattering, CRS techniques provide signal enhancement by many orders of magnitude, dramatically improving the data acquisition speed. They make use of two synchronized trains of laser pulses at frequencies $\omega_{\text {pu }}$ (pump pulse) and $\omega_{S}$ (Stokes pulse), with their frequency difference matching a characteristic vibrational frequency $\Omega$ of the molecule $\left(\omega_{\mathrm{pu}}-\omega_{S}=\Omega\right)$. CRS processes can be regarded as four-field interactions mediated by the third-order nonlinear susceptibility of the medium, $\chi^{(3)}=\chi_{N R}^{(3)}+\chi_{R}^{(3)}$, which is made up by the sum of two terms: a nonresonant real and frequencyindependent term $\chi_{N R}^{(3)}$, and a resonant complex term $\chi_{R}^{(3)}$, which, for a single vibrational mode $\Omega$, assumes the form

$$
\chi_{R}^{(3)}\left(\omega_{\mathrm{pu}}-\omega_{S}\right) \propto \frac{N \sigma}{\omega_{\mathrm{pu}}-\omega_{S}-\Omega-i \Gamma},
$$

where $N$ is the volume concentration of Raman active scatterers, $\sigma$ is the Raman cross section of the transition, and $\Gamma$ the linewidth parameter. Each CRS technique is characterized by a specific dependence on $\chi^{(3)}$.

Coherent anti-Stokes Raman scattering (CARS) [7] has been until recently the most popular form of CRS. CARS has the advantage of generating a signal at a frequency $\omega_{\mathrm{aS}}=$ $\omega_{\mathrm{pu}}+\Omega$, different with respect to those of pump and Stokes, which can be easily singled out by spectral filtering, allowing the measurement of the signal against a zero linear background [8]. However, since the CARS signal scales as $\left|\chi^{(3)}\right|^{2}$, the interplay between $\chi_{R}^{(3)}$ and $\chi_{N R}^{(3)}$ gives rise to the so-called nonresonant background (NRB), which can significantly distort or even completely overwhelm the resonant signal of interest. In addition, the quadratic concentration dependence of CARS makes it difficult to detect the less abundant species.
These drawbacks can be overcome by stimulated Raman scattering (SRS) [9-11], since both Stokes-field amplification [stimulated Raman gain (SRG)] and pump-field attenuation [stimulated Raman loss (SRL)] are linearly proportional to the imaginary part of $\chi^{(3)}$, suppressing the real NRB contribution. Moreover, thanks to its linear concentration dependence, SRS allows sensitive detection of even dilute species. On the other hand, the SRS signal sits on a large linear background given by the Stokes (pump) light and its extraction requires high-speed modulation transfer combined with lock-in detection [12,13], which is challenging especially at high acquisition speeds [14]. A CRS technique combining the advantages of CARS (absence of linear background) and SRS (absence of NRB, linear scaling with the species concentration) would thus be highly beneficial.

In this paper we introduce balanced-detection (BD) Ramaninduced Kerr effect (RIKE) as a powerful CRS technique. RIKE relies on the Raman-induced birefringence that occurs when the pump-Stokes frequency detuning is in resonance with a vibrational transition [15], leading to a polarization change of the Stokes (pump) field. RIKE, both in its single-frequency and multiplex versions, has been so far proposed for coherent vibrational spectroscopy [16] and microscopy $[17,18]$ in combination with detection schemes based on crossed polarizers. Here we show how a balanced detection configuration, inspired by that applied in electro-optic sampling in the $\mathrm{THz}$ domain [19], suppresses both the linear background and the NRB, combining the advantages of CARS with those of SRS. In addition, BD-RIKE enables homodyne amplification of the signal and exhibits a linear dependence on the concentration of the target molecules, as in SRS, as well as an effective cancellation of laser intensity noise. Finally, BD-RIKE allows straightforward and predetermined selection of either the imaginary or the real part of the nonlinear material response.

\section{COMPARISON OF COHERENT RAMAN SCATTERING TECHNIQUES}

To gain a physical insight into the BD-RIKE mechanism it is worth reviewing the well-assessed CARS and SRS techniques. For the case of CARS and neglecting phase mismatch terms, 


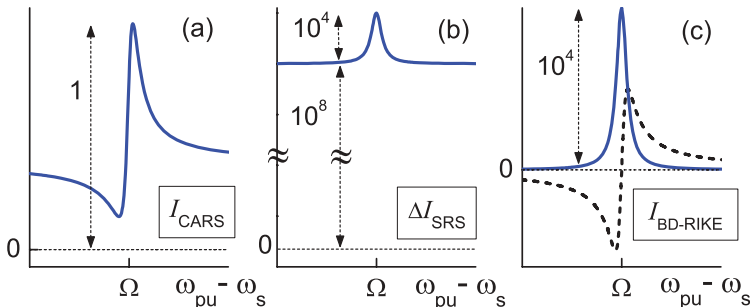

FIG. 1. (Color online) Qualitative frequency-dependence and signal amplitude of (a) CARS, (b) SRS and (c) BD-RIKE responses. The amplitudes are normalized to the CARS case. Panel (c) also highlights the capability of BD-RIKE of measuring both the imaginary (solid blue) and real (dashed black) part of the nonlinear susceptibility.

the generated field at the anti-Stokes frequency is [20] $E_{\mathrm{CARS}} \propto-i \chi^{(3)} E_{\mathrm{pu}}^{2} E_{S}^{*} L$, where $E_{\mathrm{pu}}\left(E_{S}\right)$ is the pump (Stokes) field and $L$ is the interaction length. The detected CARS signal is then given by

$$
\begin{aligned}
I_{\mathrm{CARS}} & \propto\left|E_{\mathrm{CARS}}\right|^{2} \propto\left|\chi_{R}^{(3)}+\chi_{N R}^{(3)}\right|^{2} \\
& =\left|\chi_{R}^{(3)}\right|^{2}+\left|\chi_{N R}^{(3)}\right|^{2}+2 \chi_{N R}^{(3)} \operatorname{Re}\left(\chi_{R}^{(3)}\right) .
\end{aligned}
$$

The last two terms in Eq. (2) account for the NRB. When the concentrations of the target molecules are low $\left(\chi_{R}^{(3)} \ll \chi_{N R}^{(3)}\right)$ the NRB leads to significant spectral distortion, as qualitatively shown in Fig. 1(a). In addition, due to the nonlinear scaling of the signal with concentration $\left(I_{\text {CARS }} \propto N^{2}\right)$, CARS fails to detect vibrational signatures at low concentrations of the resonant oscillators. Several techniques have been proposed to suppress the NRB, such as interferometric CARS $[21,22]$, frequency-modulation CARS [23], and time-delayed CARS $[5,24]$. However all these approaches add a significant level of technological complexity.

In the case of SRS, on the other hand, the detected signal is the Stokes field amplification (or equivalently the pump field attenuation), which amounts to $\Delta E_{S} \propto-i \chi^{(3)}\left|E_{\mathrm{pu}}\right|^{2} E_{S} L$ and sits on the large linear offset signal given by the Stokes field $E_{S}$ itself. In the limit $\Delta E_{S} \ll E_{S}$, the following expression for SRG can be calculated:

$$
\begin{aligned}
\Delta I_{\mathrm{SRS}} & \propto\left|E_{S}+\Delta E_{S}\right|^{2}-\left|E_{S}\right|^{2} \cong 2 \operatorname{Re}\left(E_{S}^{*} \Delta E_{S}\right) \\
& \propto \operatorname{Im}\left(\chi^{(3)}\right)\left|E_{\mathrm{pu}}\right|^{2}\left|E_{S}\right|^{2} L=\operatorname{Im}\left(\chi_{R}^{(3)}\right) I_{\mathrm{pu}} I_{S} L .
\end{aligned}
$$

Equation (3) highlights the main advantages of SRS with respect to CARS: (i) as the signal is proportional to the imaginary part of the nonlinear susceptibility, the real NRB does not contribute to the SRS signal; (ii) the SRG or SRL signal is directly proportional to $\chi^{(3)}$, so that it scales linearly with the sample concentration; (iii) the small nonlinear signal $\Delta E_{S}$ is multiplied by the large and phase-locked Stokes field $E_{S}$ (homodyne configuration), allowing for a large amplification of the SRS signal $\left(\approx E_{S} \Delta E_{S}\right)$ with respect to the CARS signal $\left(\approx \Delta E_{S}^{2}\right)$, as illustrated in Fig. 1(b). The main experimental drawback of SRS lies in the fact that, as in any pump-probe experiment, one needs to detect a signal sitting on top of a very large background $\left(\left|E_{S}\right|^{2}\right)$, as again evidenced in Fig. 1(b). Typical SRS signals are of the order of $10^{-4}$ or less [9]. Moreover, SRS does not allow any access to the real part of $\chi^{(3)}$.

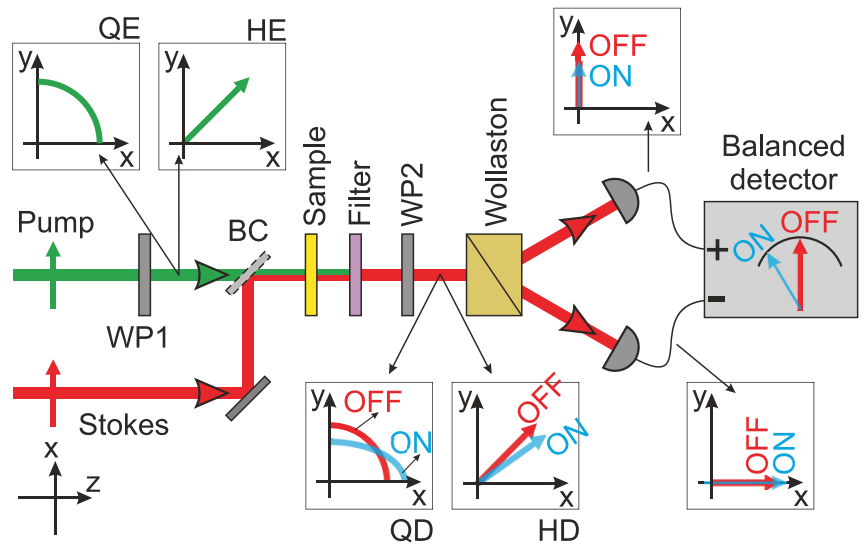

FIG. 2. (Color online) Conceptual scheme for a BD-RIKE experiment. Square panels visualize the polarization state of pump and Stokes fields in different sections of the beam path depending on the excitation and detection configuration. The polarization states of the Stokes field, since strictly related to the phase difference between $E_{s}$ and $\Delta E_{s}$, and thus to the pump-Stokes frequency detuning and to the choice of the excitation wave plate, are simplified in the figure.

\section{BALANCED-DETECTION RIKE}

A significant step forward in both directions can be obtained with BD-RIKE, the conceptual scheme of which is illustrated in Fig. 2. Experimentally, BD-RIKE is quite similar to SRS, making it easy to switch between the two configurations. Synchronized collinear pump and Stokes pulses are focused on the sample. A first wave plate (WP1) is used to control the pump polarization, and thus the excitation conditions; it can be either a quarter-wave plate [quarter-wave excitation (QE)] or a half-wave plate [half-wave excitation (HE)]. Behind the sample, the linearly polarized Stokes field is spectrally selected by a band pass filter and passes through a second wave plate (WP2), which can in turn be a quarter-wave plate [quarter-wave detection (QD)] or a half-wave plate [half-wave detection (HD)]. The transmitted beam is then sent to a Wollaston prism, which separates it into two equally intense replicas with perpendicular polarizations, followed by a balanced detector, which provides zero output in the absence of the pump field.

Changing the excitation conditions results in different terms for the nonlinear polarization, according to the expressions [17],

$$
\begin{aligned}
& P^{(3)}=\left(\chi_{1122}^{(3)}+\chi_{1212}^{(3)}\right)\left|E_{\mathrm{pu}}\right|^{2} E_{S}, \\
& P^{(3)}=i\left(\chi_{1122}^{(3)}-\chi_{1212}^{(3)}\right)\left|E_{\mathrm{pu}}\right|^{2} E_{S},
\end{aligned}
$$

for HE and QE configurations respectively. $\chi_{1122}^{(3)}$ and $\chi_{1212}^{(3)}$ are nondiagonal terms of the third-order nonlinear susceptibility tensor, including both resonant and nonresonant contributions. Since, due to the Kleinmann symmetry [25],

$$
\chi_{1122 N R}^{(3)}=\chi_{1212 N R}^{(3)}=\frac{1}{3} \chi_{1111 N R}^{(3)}
$$

the nonresonant contribution to the third-order nonlinear vanishes in the $\mathrm{QE}$ configuration.

Equations (4) allow the RIKE-induced modulation of the Stokes field to be evaluated for both HE configuration [26]

$$
\Delta E_{S} \propto-i P^{(3)} L=-i\left(\chi_{1122}^{(3)}+\chi_{1212}^{(3)}\right)\left|E_{\mathrm{pu}}\right|^{2} E_{S} L
$$


TABLE I. Comparison of BD-RIKE coefficient $\eta$ using different combinations of half- and quarter-wave plates in excitation and detection.

\begin{tabular}{lll}
\hline \hline & \multicolumn{2}{c}{ Detection } \\
\cline { 2 - 3 } & QD & HD
\end{tabular}

\begin{tabular}{ccc}
\hline Excitation & \\
$\mathrm{HE}$ & $\operatorname{Re}\left[\begin{array}{l}\chi_{1122 R}^{(3)}+\chi_{1212 R}^{(3)}+ \\
\chi_{1122 N R}^{(3)}+\chi_{1212 N R}^{(3)}\end{array}\right]$ & $\operatorname{Im}\left[\chi_{1122 R}^{(3)}+\chi_{1212 R}^{(3)}\right]$ \\
$\mathrm{QE}$ & $\operatorname{Im}\left[\chi_{1122 R}^{(3)}-\chi_{1212 R}^{(3)}\right]$ & $\operatorname{Re}\left[\chi_{1122 R}^{(3)}-\chi_{1212 R}^{(3)}\right]$ \\
\hline \hline
\end{tabular}

and $\mathrm{QE}$ configuration

$$
\Delta E_{S} \propto-i P^{(3)} L=\left(\chi_{1122}^{(3)}-\chi_{1212}^{(3)}\right)\left|E_{\mathrm{pu}}\right|^{2} E_{S} L,
$$

the polarization of the nonlinear field $\Delta E_{s}$ being now orthogonal to that of the Stokes field $E_{s}$. Equations (6) evidence that the phase relation between $E_{s}$ and $\Delta E_{S}$, and thus the polarization state of the Stokes field after the sample, varies depending on the excitation conditions and also on the pump-Stokes frequency detuning, due to the complex nature of the $\chi^{(3)}$ terms. When reading such polarization state with a detection chain composed of WP2, Wollaston prism and balanced detector, the photocurrent is found to be proportional to the following intensity terms:

$$
\begin{aligned}
& I_{\mathrm{BD}-\mathrm{RIKE}} \propto 2 \operatorname{Re}\left(E_{S} \Delta E_{S}^{*}\right) \\
& I_{\mathrm{BD}-\mathrm{RIKE}} \propto 2 \operatorname{Im}\left(E_{S} \Delta E_{S}^{*}\right)
\end{aligned}
$$

for HD and QD configurations respectively [see Appendix for analytical derivation of Eqs. (7)]. The two detection conditions set by Eqs. (7), when combined with the two excitation conditions set by Eqs. (6), give rise to a BD-RIKE signal $I_{\mathrm{BD}-\mathrm{RIKE}} \propto \eta I_{\mathrm{pu}} I_{S} L$ with $\eta$ depending on the four different BD-RIKE regimes. Each configuration addresses a specific term of the third-order susceptibility of the sample, as summarized in Table I.

We are now in a position to compare BD-RIKE with the more established CARS and SRS techniques.

(i) Thanks to balanced detection, BD-RIKE removes the common-mode Stokes (pump) contribution and allows one to measure the nonlinear signal against a zero linear background [see Fig. 1(c)].

(ii) Similarly to SRS, it inherently provides homodyne amplification of the nonlinear signal [see Eqs. (7)], increasing its magnitude by many orders of magnitude [see Fig. 1(c)] .

(iii) The BD-RIKE signal is proportional to $\chi^{(3)}$, thus scaling linearly with the sample concentration, as in SRS, and allowing a straightforward quantitative analysis of the spectra and the images.

(iv) In three out of four configurations (QE-QD, QE-HD, and HE-HD) it suppresses NRB, allowing a purely resonant susceptibility to be measured.

(v) By properly choosing the excitation and detection conditions it allows, to the user's choice, to address either the real or the imaginary part of the nonlinear susceptibility [see solid and dashed lines in Fig. 1(c)]. (vi) Due to the balanced detection, laser intensity noise on the Stokes (pump) pulse is efficiently suppressed, thus favouring shot-noise limited detection.

\section{RESULTS}

We experimentally tested BD-RIKE using a 40-MHz Erdoped fiber oscillator with two amplified branches (Toptica, FemtoPro model), both delivering nearly $\approx 70$-fs long pulses at $1550 \mathrm{~nm}$ and $350 \mathrm{~mW}$ average power. One of the outputs is coupled to a highly nonlinear fiber producing an octavespanning supercontinuum (SC), the long-wavelength part of which is tunable from 1700 to $2100 \mathrm{~nm}$. Narrow band pump $\left(10 \mathrm{~cm}^{-1}\right)$ and Stokes $\left(30 \mathrm{~cm}^{-1}\right)$ pulses are respectively generated by spectral compression of the $1550-\mathrm{nm}$ pulse and of the SC in periodically poled lithium niobate (PPLN) crystals [26-29]. Available power outputs are $100 \mathrm{~mW}$ for the pump and $10 \mathrm{~mW}$ for the Stokes beam. The pump pulses are fixed at $780 \mathrm{~nm}$, while the Stokes wavelength can be continuously tuned using a fan-out PPLN crystal. Pump and Stokes pulses are combined by a dichroic beam splitter and focused onto the sample. The pump beam is acousto-optically modulated and the BD-RIKE signal is detected by a lock-in amplifier in order to remove any contributions by the static birefringence of the sample. In this way the measurement is sensitive only to the dynamic birefringence induced by the pump beam.

Figure 3 shows BD-RIKE spectra of acetone acquired under the four possible excitation and detection configurations, together with curves calculated according to the expressions given in Table I, simultaneously fitting the four experimental data with a unique set of parameters. The fits are in good agreement with the measurements, with small deviations being mostly attributed to imperfect phase retardation given by the wave plates at the working wavelengths. The data demonstrate the capability of BD-RIKE of effectively suppressing NRB (except for the HE-QD configuration) and measuring either the bell-shaped imaginary (QE-QD, HE-HD) or the dispersive real (QE-HD) component of the nonlinear susceptibility.

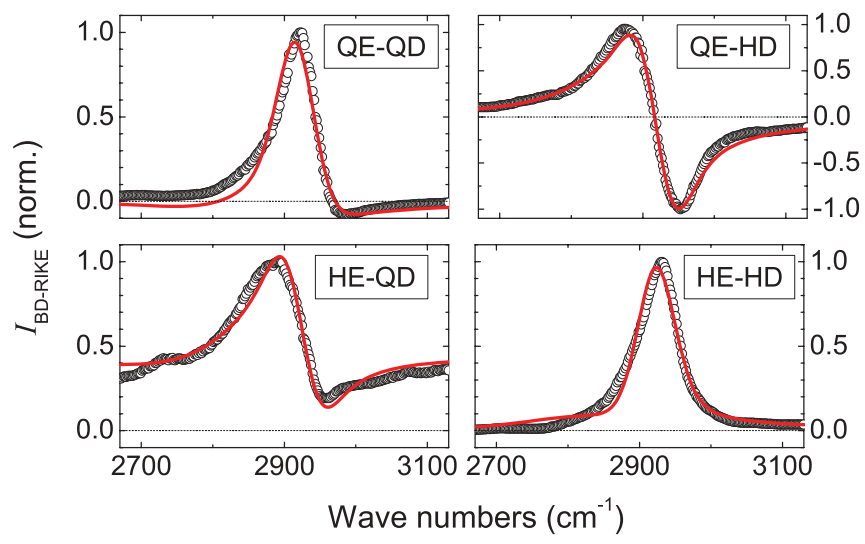

FIG. 3. (Color online) BD-RIKE spectra of acetone under different excitation and detection conditions (circles) and numerical fits with the expressions reported in Table I (solid lines). The fits used for the four measurements a single set of parameters with a dominant Raman mode at $\Omega=2922 \mathrm{~cm}^{-1}\left(\Gamma=9.8 \mathrm{~cm}^{-1}\right)$ and a weaker mode at $\Omega=2815 \mathrm{~cm}^{-1}\left(\Gamma=100 \mathrm{~cm}^{-1}\right)$. The fits assumed an instrumental resolution of $31.5 \mathrm{~cm}^{-1}$. 


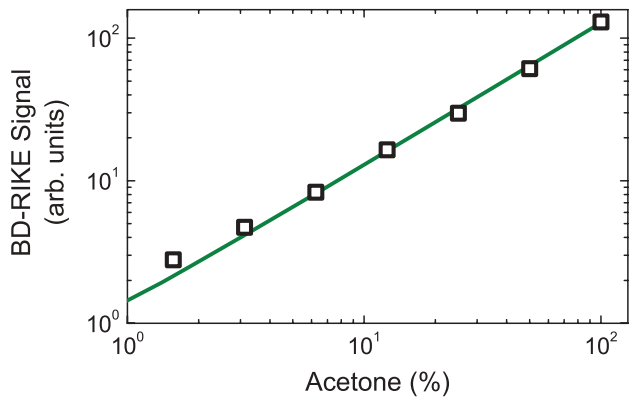

FIG. 4. (Color online) Dilution test of acetone in water acquired in the HE-HD configuration, displaying linear concentration dependence of the BD-RIKE signal.

Inspecting Table I, the most favourable configuration giving the highest signal is the HE-HD, as also confirmed by experiments. For this configuration Fig. 4 shows the result of a dilution test of acetone in water displaying a linear dependence on sample concentration down to $1 \%$, as expected from the self-homodyning capabilities of BD-RIKE. Note that this value does not represent the lowest detection limit, but rather the value at which the nonlinear Raman signal from water becomes comparable to that from acetone.

To demonstrate the inherent noise rejection capabilities of BD-RIKE, Fig. 5 shows a direct comparison between BD-RIKE images in the HE-HD configuration and SRS images, as acquired under the same experimental conditions. As a sample we chose a blend of $3-\mu \mathrm{m}$ polystyrene (PS)

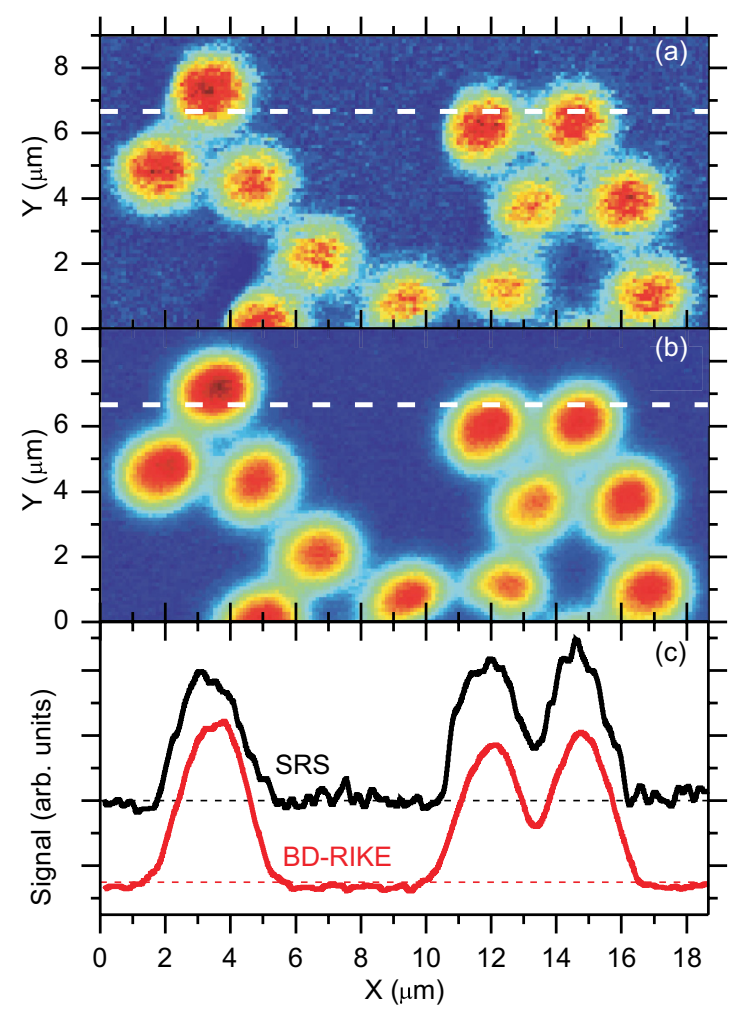

FIG. 5. (Color online) (a) SRS and (b) HE-HD BD-RIKE images of a blend of 3- $\mu \mathrm{m}$ PS and 6- $\mu \mathrm{m}$ PMMA beads; (c) horizontal cuts along the dotted lines. and 6- $\mu \mathrm{m}$ poly-methylmethacrylate beads and probed it at the $3060 \mathrm{~cm}^{-1}$ PS resonance, using $\approx 10 \mathrm{~mW}$ pump power, $1-\mathrm{mW}$ Stokes power and low modulation frequency $(40 \mathrm{kHz})$. The BD-RIKE images [Fig. 5(b)] display a significantly better quality compared to the SRS ones [Fig. 5(a)], as confirmed by the intensity profiles along the dotted lines, shown in Fig. 5(c). This improvement is due to the inherent commonmode rejection of the laser intensity noise offered by balanced detection, and becomes particularly important when working under non-shot-noise limited conditions, as in the present case. For a sample with strong and heterogeneous birefringence, the noise rejection capability of BD-RIKE would be mitigated, due to nonperfect balanced detection.

\section{CONCLUSION}

In conclusion, we have introduced a CRS technique that sensitively measures the RIKE-induced polarization rotation using a balanced detection configuration. BD-RIKE presents an unprecedented mix of features, namely absence of both linear and nonlinear background, homodyne amplification of the nonlinear Raman signal, linear dependence on the sample concentration, absence of NRB, as well as the possibility, to the user's choice, to address either the real or the imaginary part of $\chi^{(3)}$, thus making it possible to obtain quite sophisticated molecular responses for enhanced chemical selectivity. BDRIKE is a valuable addition to the portfolio of CRS techniques, which can be seamlessly integrated in existing CRS setups with minimal technological effort and the potential to enhance their performance for several biomedical or nanotechnological imaging and spectroscopy applications.

\section{ACKNOWLEDGMENTS}

The authors acknowledge support by the EU FP7 FET Project CROSS TRAP (Contract No. ICT-244068) and by the project of Regione Lombardia (CUP No. D41J10000300007).

\section{APPENDIX: ANALYSIS OF THE RESPONSE OF THE BD-RIKE DETECTION CHAIN}

The BD scheme described in the paper is inspired by the basic technique traditionally used in electro-optic sampling to detect the polarization rotation of a gate pulse induced by the THz field. Its main building blocks are a wave plate, a Wollaston prism, and two balanced photodetectors.

We will first illustrate graphically the working principle of this arrangement in the particular case in which it is used to detect a change in the polarization of a field, as depicted in Fig. 6. This example employs a half-wave plate, which is initially configured so that the unperturbed field (corresponding in BD-RIKE to the Stokes field under a pump-off condition) equally projects along the $x$ and $y$ axis [see Fig. 6(a)]. When the polarization of the field is rotated (pump-on state in BD-RIKE), $x$ and $y$ projections become unbalanced, giving rise to a finite differential signal [Fig. 6(b)], whose sign is related to the rotation direction.

In a more general case the aim is to detect an arbitrary polarization change of a given field initially polarized along the $x$ axis under the action of a perturbation that introduces 

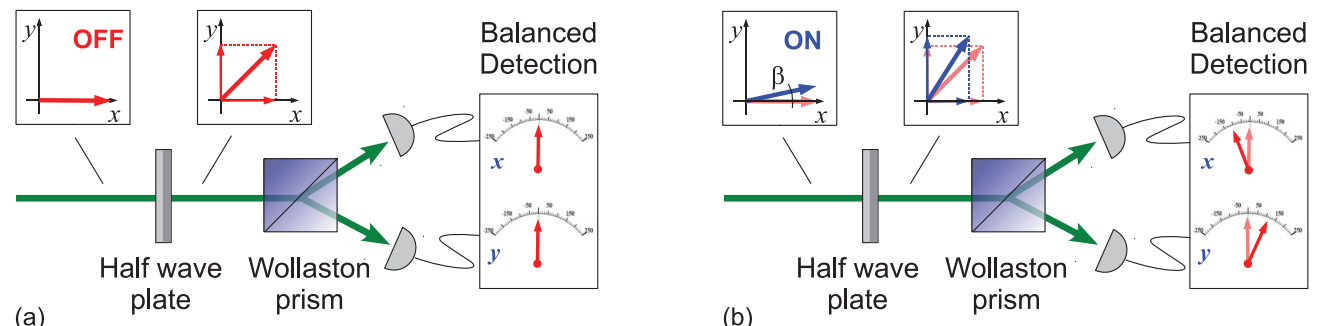

FIG. 6. (Color online) Conceptual working principle of the BD scheme for the detection of a polarization rotation in the (a) unperturbed and (b) perturbed case.

a non-negligible component along the $y$ axis. Such component can either be in phase with the $x$ component (the polarization rotates but remains linear) or out of phase (the polarization becomes elliptical). The detection can make use either of a HWP or of a QWP. Depending on the wave plate, the photocurrent produced at the output of the balanced detector is proportional either to the real part of the perturbed field (in phase with the unperturbed field) or to the imaginary part of it (in quadrature with the unperturbed field). The two configurations thus provide complementary information, and a full reconstruction of the perturbation is possible only whenever are both implemented. Let us indicate with $E$ a field propagating along the $z$ direction and with $E_{x}$ and $E_{y}$ its components along the polarization axes of the Wollaston prism, according to the scheme reported in Fig. 7,

$$
\begin{gathered}
E_{x}=A_{x} e^{j \omega t} \\
E_{y}=A_{y} e^{j \omega t} .
\end{gathered}
$$

Here $\omega$ is the carrier frequency, $A_{x}$ and $A_{y}$ are fields complex amplitudes, $A_{x}$ being the unperturbed reference field, and $A_{y}$ the signal field to be detected. Let us now treat separately the case of a half- and quarter-wave plate detection by indicating with $\alpha$ the angle by which the axis of the wave plate is rotated with respect to $x$, as indicated in Fig. 7 .

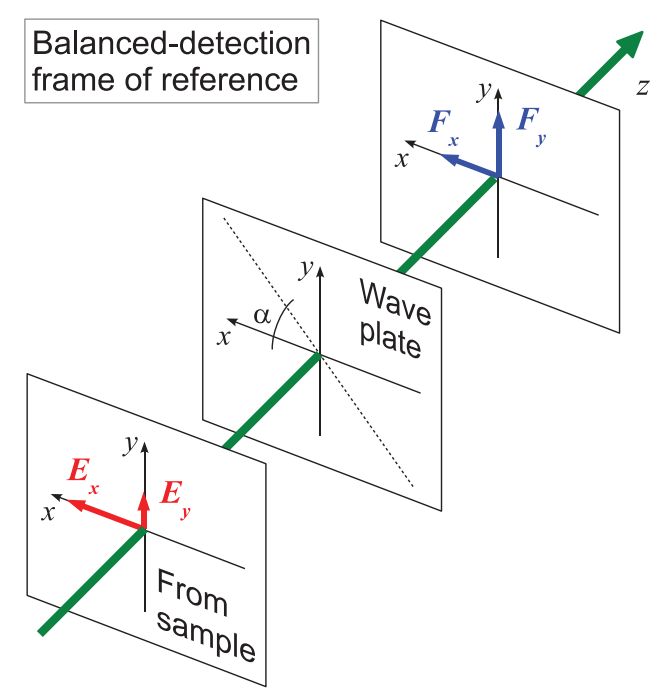

FIG. 7. (Color online) Reference frame of the balanced detection scheme. The rotation angle of the half- or quarter-wave plate is also indicated.

\section{Detection scheme with half-wave plate}

In the unperturbed regime, where only $E_{x}$ is propagating through the system, the transmitted field $F_{0}$ has components

$$
\begin{aligned}
& F_{0 x}=A_{x}(\cos 2 \alpha) e^{j \omega t} \\
& F_{0 y}=A_{x}(\sin 2 \alpha) e^{j \omega t} .
\end{aligned}
$$

Their average intensity is

$$
\begin{aligned}
& \left\langle\left|F_{0 x}\right|^{2}\right\rangle=\left|A_{x}\right|^{2} \cos ^{2} 2 \alpha \\
& \left\langle\left|F_{0 y}\right|^{2}\right\rangle=\left|A_{x}\right|^{2} \sin ^{2} 2 \alpha .
\end{aligned}
$$

To get perfectly balanced signals, the HWP has to be rotated by $\alpha=\pi / 8+k \pi / 4$, which corresponds to rotating the linear polarization of $F_{0}$ at an angle of $\pi / 4+k \pi / 2$. Since the physics of the problem is invariant with respect to $k$, we will hereafter consider the case $\alpha=\pi / 8$. At this rotation of the HWP, we are now in a position to evaluate the transmitted total field $F$ in the presence of a perturbation $E_{y}$. The resulting components of $F$ are

$$
\begin{gathered}
F_{x}=\frac{\sqrt{2}}{2}\left[A_{x}+A_{y}\right] e^{j \omega t} \\
F_{y}=\frac{\sqrt{2}}{2}\left[A_{x}-A_{y}\right] e^{j \omega t} .
\end{gathered}
$$

The corresponding average intensities of the $x$ and $y$ components separated by the Wollaston prism and detected by the balanced photodiodes are

$$
\begin{aligned}
\left\langle\left|F_{x}\right|^{2}\right\rangle & =\frac{\sqrt{2}}{2}\left[A_{x}+A_{y}\right] \frac{\sqrt{2}}{2}\left[A_{x}+A_{y}\right]^{*} \\
& =\frac{1}{2}\left[\left|A_{x}\right|^{2}+\left|A_{y}\right|^{2}+2 \operatorname{Re}\left(A_{x} A_{y}^{*}\right)\right] \\
\left\langle\left|F_{y}\right|^{2}\right\rangle & =\frac{\sqrt{2}}{2}\left[A_{x}-A_{y}\right] \frac{\sqrt{2}}{2}\left[A_{x}-A_{y}\right]^{*} \\
& =\frac{1}{2}\left[\left|A_{x}\right|^{2}+\left|A_{y}\right|^{2}-2 \operatorname{Re}\left(A_{x} A_{y}^{*}\right)\right],
\end{aligned}
$$

where $A_{x}^{*}$ is the complex conjugate of signal field $A_{x}$. Since the balanced detection extracts $\left\langle\left|F_{x}\right|^{2}\right\rangle-\left\langle\left|F_{y}\right|^{2}\right\rangle$ the differential signal results to be

$$
\left\langle\left|F_{x}\right|^{2}\right\rangle-\left\langle\left|F_{y}\right|^{2}\right\rangle=2 \operatorname{Re}\left(A_{x} A_{y}^{*}\right),
$$

proportional to the real part of the product $A_{x} A_{y}^{*}$. 


\section{Detection scheme with quarter-wave plate}

In the case of QWP detection, the components of the transmitted field $F_{0}$ are

$$
\begin{aligned}
& F_{0 x}=A_{x}\left(\cos ^{2} \alpha+e^{j \pi / 2} \sin ^{2} \alpha\right) e^{j \omega t} \\
& F_{0 y}=A_{x}\left(1-e^{j \pi / 2}\right) \sin \alpha \cos \alpha e^{j \omega t},
\end{aligned}
$$

corresponding to an average intensity

$$
\begin{aligned}
& \left\langle\left|F_{0 x}\right|^{2}\right\rangle=\left|A_{x}\right|^{2}\left(\cos ^{4} \alpha+\sin ^{4} \alpha\right) \\
& \left\langle\left|F_{0 y}\right|^{2}\right\rangle=\left|A_{x}\right|^{2} 2 \sin ^{2} \alpha \cos ^{2} \alpha .
\end{aligned}
$$

As expected, balanced detection is obtained when $\alpha=\pi / 4$ and $\alpha=3 \pi / 4$; both angles give a circularly polarized $F$ field. In the following we will focus on the case $\alpha=\pi / 4$ since the $\alpha=3 \pi / 4$ case leads to the same results. In the presence of the perturbing field $E_{y}$ the components of the transmitted field $F$ after the QWP are

$$
\begin{aligned}
& F_{x}=\frac{1}{2}\left[A_{x}(1+j)+A_{y}(1-j)\right] e^{j \omega t} \\
& F_{y}=\frac{1}{2}\left[A_{x}(1-j)+A_{y}(1+j)\right] e^{j \omega t} .
\end{aligned}
$$

The corresponding average intensities along $x$ and $y$ detected by the balanced photodiodes are

$$
\begin{aligned}
\left\langle\left|F_{x}\right|^{2}\right\rangle & =\frac{1}{4}\left[A_{x}(1+j)+A_{y}(1-j)\right]\left[A_{x}(1+\mid, j)+A_{y}(1-j)\right]^{*} \\
& =\frac{1}{2}\left[\left|A_{x}\right|^{2}+\left|A_{y}\right|^{2}-2 \operatorname{Im}\left(A_{x} A_{y}^{*}\right)\right] \\
\left\langle\left|F_{y}\right|^{2}\right\rangle & =\frac{1}{2}\left[A_{x}(1-j)+A_{y}(1+j)\right]\left[A_{x}(1-j)+A_{y}(1+j)\right]^{*} \\
& =\frac{1}{2}\left[\left|A_{x}\right|^{2}+\left|A_{y}\right|^{2}+2 \operatorname{Im}\left(A_{x} A_{y}^{*}\right)\right]
\end{aligned}
$$

and the differential signal given by balanced detection is

$$
\left\langle\left|F_{y}\right|^{2}\right\rangle-\left\langle\left|F_{x}\right|^{2}\right\rangle=2 \operatorname{Im}\left(A_{x} A_{y}^{*}\right),
$$

which is proportional to the imaginary part of the product $A_{x} A_{y}^{*}$. The use of HWP and QWP is therefore complementary, and allows extracting the real and imaginary part of $A_{x} A_{y}^{*}$.

Inspection of Eqs. (A6) and (A11) shows that balanced detection returns the product between the gate field $A_{x}$ and the perturbation $A_{y}$. This capability results in the following properties. (i) The use of a QWP or a HWP allows to measure either $\operatorname{Re}\left(A_{x} A_{y}^{*}\right)$ or $\operatorname{Im}\left(A_{x} A_{y}^{*}\right)$. This gives the unique possibility to get access to all information on the complex field $A_{y}$.

(ii) The technique benefits from the typical advantages of homodyne detection, in which the signal is enhanced by a copropagating field at the same carrier frequency. Here the product amplifies the perturbation $A_{y}$ by many orders of magnitude, thus allowing to extract even very weak $A_{y}$ fields.

(iii) The measurement is background free, since the differential signal is zero when there is no signal $A_{y}$.

\section{Application to the measurement of polarization rotations}

As a representative example let us now consider the analytical description of the case qualitatively described above with reference to Fig. 6 (i.e., the measurement of a linear polarization rotation). If the field is rotated by an angle $\beta$, the electric field components of the beam impinging the detection wave plate can be written as

$$
\begin{aligned}
& E_{x}=A_{0} \cos \beta e^{j \omega t} \\
& E_{y}=A_{0} \sin \beta e^{j \omega t} .
\end{aligned}
$$

If we now apply a BD scheme with HWP, according to Eq. (A6) we get the following differential signal:

$$
2 \Re\left(A_{x} A_{y}^{*}\right)=2\left|A_{0}\right|^{2} \sin \beta \cos \beta=\left|A_{0}\right|^{2} \sin 2 \beta,
$$

which, for small rotations, is proportional to the angle $\beta$. Differently, when adopting a traditional technique making use of a polarizer to extract the intensity of the $y$ component only, the average transmitted intensity would be

$$
\left\langle\left|E_{y}\right|^{2}\right\rangle=\left|A_{0}\right|^{2} \sin ^{2} \beta
$$

proportional to the square of $\beta$. This example highlights the potential of a BD scheme for the detection of small polarization rotations.

If adopting a HWP detection scheme to the same case, no differential signal would be observed at the output of the balanced detector, the perturbed field being in phase with the reference field.
[1] G. L. Eesley, J. Quant. Spectrosc. Radiat. Transfer 22, 507 (1979).

[2] C. L. Evans, E. O. Potma, M. Puoris'haag, D. Cote, C. P. Lin, and X. S. Xie, Proc. Natl. Acad. Sci. USA 102, 16807 (2005).

[3] T. Hellerer, C. Axäng, Ch Brackmann, P. Hillertz, M. Pilon, and A. Enejder, Proc. Natl. Acad. Sci. USA 104, 14658 (2007).

[4] C. L. Evans and X. S. Xie, Annu. Rev. Anal. Chem. 1, 883 (2008).

[5] C. Pohling, T. Buckup, A. Pagenstecher, and M. Motzkus, Biomed. Opt. Express 2, 2110 (2011).

[6] D. Pestov, R. K. Murawski, G. O. Ariunbold, X. Wang, M. C. Zhi, A. V. Sokolov, V. A. Sautenkov, Y. V. Rostovtsev, A. Dogariu, Y. Huang, and M. O. Scully, Science 316, 265 (2007).

[7] R. F. Begley, A. B. Harvey, and R. L. Byer, Appl. Phys. Lett. 25, 387 (1974).

[8] A. Zumbusch, G. R. Holtom and X. S. Xie, Phys. Rev. Lett. 82, 4142 (1999).
[9] A. Owyoung and E. D. Jones, Opt. Lett. 1, 152 (1977).

[10] Ch.W. Freudiger, W. Min, B. G. Saar, S. Lu, G. R. Holtom, C. He, J. C. Tsai, J. X. Kang, and X. S. Xie, Science 322, 1857 (2008).

[11] P. Nandakumar, A. Kovalev, and A. Volkmer, New J. Phys. 11, 033026 (2009).

[12] Y. Ozeki, F. Dake, S. Kajiyama, K. Fukui, and K. Itoh, Opt. Express 17, 3651 (2009).

[13] B. G. Saar, Ch. W. Freudiger, J. Reichman, C. M. Stanley, G. R. Holtom, and X. S. Xie, Science 330, 1368 (2010).

[14] J. P. Pezacki, J. A. Blake, D. C. Danielson, D. C. Kennedy, R. K. Lyn, and R. Singaravelu, Nature Chem. Biol. 7, 137 (2011).

[15] D. Heiman, R. W. Hellwarth, M. D. Levenson, and G. Martin, Phys. Rev. Lett. 36, 189 (1976).

[16] S. Shim and R. A. Mathies, J. Raman Spectrosc. 39, 1526 (2008).

[17] C. W. Freudiger, M. B. J. Roeffaers, X. Zhang, B. G. Saar, W. Min, and X. S. Xie, J. Phys. Chem. B 115, 5574 (2011). 
[18] B. R. Bachler, M. E. Fermann, and J. P. Ogilvie, Opt. Express 20, 835 (2012)

[19] Q. Wu and X.-C. Zhang, Appl. Phys. Lett. 71, 1285 (1997).

[20] R. W. Boyd, Nonlinear Optics, 3rd ed. (Academic Press, Boston, 2008).

[21] M. Jurna, J. P. Korterik, C. Otto, J. L. Herek, and H. L. Offerhaus, Opt. Express 16, 15863 (2008).

[22] M. Jurna, J. P. Korterik, C. Otto, J. L. Herek, and H. L. Offerhaus, Phys. Rev. Lett. 103, 043905 (2009).

[23] F. Ganikhanov, C. L. Evans, B. G. Saar, and X. S. Xie, Opt. Lett. 31, 1872 (2006).

[24] R. Selm, M. Winterhalder, A. Zumbusch, G. Krauß, T. Hanke, A. Sell, and A. Leitenstorfer, Opt. Lett. 35, 3282 (2010).
[25] M. D. Levenson and S. S. Kano, Introduction to Nonlinear Laser Spectroscopy (Academic Press, San Diego, 1988).

[26] S. Mukamel, Principles of Nonlinear Optical Spectroscopy (Oxford University Press, New York, 1995).

[27] M. Marangoni, A. Gambetta, C. Manzoni, V. Kumar, R. Ramponi, and G. Cerullo, Opt. Lett. 34, 3262 (2009).

[28] G. Krauss, T. Hanke, A. Sell, D. Träutlein, A. Leitenstorfer, R. Selm, M. Winterhalder, and A. Zumbusch, Opt. Lett. 34, 2847 (2009).

[29] A. Gambetta, V. Kumar, G. Grancini, D. Polli, R. Ramponi, G. Cerullo, and M. Marangoni, Opt. Lett. 35, 226 (2010). 